\title{
Survival Nomograms after Curative Neoadjuvant Chemotherapy and Radical Surgery for Stage IB2-IIIB Cervical Cancer
}

\author{
Claudia Marchetti, $\mathrm{PhD}$ \\ Francesca De Felice, $\mathrm{PhD}^{2}$ \\ Anna Di Pinto, MD \\ Alessia Romito, MD' \\ Angela Musella, MD \\ Innocenza Palaia, $\mathrm{PhD}^{1}$ \\ Marco Monti, MD ${ }^{1}$ \\ Vincenzo Tombolini, FP2 \\ Ludovico Muzii, $\mathrm{PhD}^{1}$ \\ PierLuigi Benedetti Panici, FP1
}

Departments of ${ }^{1}$ Gynecological and Obstetrical Sciences and Urological Sciences and ${ }^{2}$ Radiotherapy, Policlinico Umberto

I "Sapienza" University of Rome, Rome, Italy

Correspondence: Claudia Marchetti, PhD Department of Gynecological and Obstetrical Sciences and Urological Sciences, University of Rome "Sapienza", Viale del Policlinico 155,

00161 Rome, Italy

Tel: 39-0649973411

Fax: 39-0649973411

E-mail: clamarchetti@libero.it

Received March 20, 2017

Accepted July 17, 2017

Published Online July 19, 2017

${ }^{*}$ Claudia Marchetti and Francesca De Felice contributed equally to this work.

\begin{abstract}
Purpose
The purpose of this study was to develop nomograms for predicting the probability of overall survival (OS) and progression-free survival (PFS) in locally advanced cervical cancer treated with neoadjuvant chemotherapy and radical surgery.
\end{abstract}

\begin{abstract}
Materials and Methods
Nomograms to predict the 5-year OS rates and the 2-year PFS rates were constructed. Calibration plots were constructed, and concordance indices were calculated. Evaluated variables were body mass index, age, tumor size, tumor histology, grading, lymphovascular space invasion, positive parametria, and positive lymph nodes.

\section{Results}

In total 245 patients with locally advanced cervical cancer who underwent neoadjuvant chemotherapy and radical surgery were included for the construction of the nomogram. The 5-year OS and PFS were $72.6 \%$ and $66 \%$, respectively. Tumor size, grading, and parametria status affected the rate of OS, whereas tumor size and positive parametria were the main independent PFS prognostic factors.
\end{abstract}

\section{Conclusion}

We constructed a nomogram based on clinicopathological features in order to predict 2-year PFS and 5-year OS in locally advanced cervical cancer primarily treated with neoadjuvant chemotherapy followed by radical surgery. This tool might be particularly helpful for assisting in the follow-up of cervical cancer patients who have not undergone concurrent chemoradiotherapy.

\section{Introduction}

Cervical cancer is the third most commonly diagnosed cancer and the fourth leading cause of cancer death in females, even if in some countries, such as Asia, the absolute number of cervical cancer cases has decreased from 4,443 in 1999 to 3,857 in 2010 [1,2].

It is well established that concurrent chemoradiation (CRT), as well as neoadjuvant chemotherapy (NACT) fol-

\section{Key words}

Nomograms, Locally advanced disease,

Uterine cervical neoplasms, Neoadjuvant chemotherapy,

Radical surgery, Clinical outcomes, Survival, Recurrence lowed by radical surgery (RS), are both superior to radiotherapy alone in terms of overall survival (OS), in locally advanced cervical cancer (LACC) management [3,4]. But several controversies still exist to identify the more appropriate strategy. Tumor down staging, improved resectability and potential beneficial effects on micrometastasis encourage the use of a NACT approach in the management of this disease [5]. In the era of individualized cancer treatment, the use of prediction model to estimate survival outcomes would be particularly helpful in clinical decision-making. Different 
prognostic models are available in LACC patients receiving CRT [6-10]. Whereas, a prognostic predictor for LACC patients treated with NACT plus RS has not yet been implemented and validated.

The aim of the current study was to find clinicopathologic variables associated with OS and progression-free survival (PFS) following curative NACT and RS for LACC, and create a nomogram for individual risk prediction.

\section{Materials and Methods}

\section{Study population}

Clinical data of cervical cancer patients undergoing NACT and surgical curative resection between January 2004 and December 2011 were retrospectively collected from our dataset. Patients with distant metastases at diagnosis, unoperated, with history of previous pelvic radiation therapy or chemotherapy and those with missing data were excluded. All patients were treated with a multimodal treatment approach, including platinum-based chemotherapy and surgery, scheduled 5-7 weeks after the end of NACT, independently of clinical response. The NACT regimen was not predetermined, but minimal requirements were a cisplatincontaining regimen with a $225 \mathrm{mg} / \mathrm{m}^{2}$ total cisplatin dose with a maximum of two additional drugs, administered over a period of 6 to 8 weeks.

Before NACT, all patients were submitted to a gynecological examination under general anesthesia. A total body computed tomography $(\mathrm{CT})$ scan or magnetic resonance imaging (MRI) was performed in all patients. Cystoscopy was performed in patients with suspected advanced disease in the anterior parametria at gynecological examination or having a suspicious invasion on CT scan or MRI. After three cycles of NACT, patients were clinically reassessed and classified as suitable or unsuitable for RS. RS consisted of systematic (at least 20 nodes to be resected) pelvic lymphadenectomy plus radical hysterectomy (type III to V) plus. Aortic lymphadenectomy was reserved for patients with pelvic node disease at intraoperative examination or finding of bulky aortic nodes at preoperative imaging or at time of surgery. If required, additional surgical procedures were performed: ureteral, full thickness and extramucosal bladder resection, uretero-ureteral anastomosis, or ureteral reimplantation. Patients not considered amenable of radical surgery after chemotherapy treatment were excluded from our analysis (intention per protocol analysis) as well as patients whose physical condition made optimal surgery unsafe (Eastern Cooperative Oncology Group performance status 2 after
NACT; serious comorbidities). Patients deemed inoperable were offered chemoradiotherapy or palliative chemotherapy.

After radical surgery, all specimens were histopathologically reviewed, and the pathological TNM class and stage were determined according to the classification established by the American Joint Committee on Cancer, seventh edition [11]. Patients staged with a prior edition staging system were restaged according to the seventh edition. According to institutional policy, adjuvant treatment, such as chemotherapy and/or radiotherapy, was recommended in those patients with pathologic risk factors detected in the operative specimen, including positive vaginal margin, positive parametrial margin, and pelvic or aortic lymph node metastasis. Adjuvant radiotherapy was used to treat the whole pelvis, including the common iliac and periaortic lymph nodes if positive. Patients were followed according to internal protocol weekly during chemotherapy treatment and up to 2 weeks postsurgery. After treatment, patients were monitored 3 monthly intervals for the first 2 years, at 4-6 monthly intervals for the additional 2 years, and annually for subsequent years. Patients were followed up closely to detect persistent or recurrent disease by clinical examination. Imaging, positron emission tomography/CT or CT scan, was performed 12 weeks after surgery to assess disease response. We included only patients for whom at least 5-year follow-up was available, because OS nomogram reflect 5-year event rates.

\section{Statistical analysis}

Statistical analysis was carried out using R-Studio 0.98. 1091 software and SPSS ver. 24.0 (IBM Corp., Armonk, NY). Standard descriptive statistics were used to evaluate the distribution of each factor. Continuous variables were presented as medians and ranges, and dichotomous variables were presented as percentages. Student's $t$ test and Wilcoxon ranksum test were used to evaluate any differences in continuous variables; Pearson's chi-square test or Fisher exact test was used for categorical variables.

OS and PFS were calculated in months from the date of diagnosis to the first event, including date of the last followup or death (OS) and/or relapse (PFS).

The evaluated variables were age ( $<50$ vs. $\geq 50)$, smoker (no vs. yes), body mass index (BMI; $<25$ vs. $\geq 25$ ), tumor size $(<4 \mathrm{~cm}$ vs. $\geq 4 \mathrm{~cm}$ ), tumor histology (squamous cell carcinoma vs. other histologies), tumor grade (G1 vs. G2 vs. G3), International Federation of Gynecology and Obstetrics (FIGO) disease stage (IB2-IIA vs. IIB vs. III), lymphovascular space invasion (absence vs. presence), positive parametria (no vs. yes), and pathologic nodal status (negative vs. positive). Firstly, univariate analysis was carried out to assess the single variables for predictive power. Then, those variables 
Table 1. Patient characteristics

\begin{tabular}{|c|c|}
\hline Characteristic & No. $(\%)$ \\
\hline \multicolumn{2}{|l|}{ Age (yr) } \\
\hline Median (range) & $51(24-83)$ \\
\hline$<50$ & $116(47.3)$ \\
\hline$\geq 50$ & $129(52.7)$ \\
\hline \multicolumn{2}{|l|}{ Smoker } \\
\hline Yes & $56(22.9)$ \\
\hline No & $189(77.1)$ \\
\hline \multicolumn{2}{|c|}{ Body mass index $\left(\mathrm{kg} / \mathrm{m}^{2}\right)$} \\
\hline$<25$ & $165(67.3)$ \\
\hline$\geq 25$ & $80(32.7)$ \\
\hline \multicolumn{2}{|l|}{ Histology } \\
\hline Squamous & $208(84.9)$ \\
\hline Adenocarcinoma & $27(11)$ \\
\hline Adenosquamous & $10(4.1)$ \\
\hline \multicolumn{2}{|l|}{ Tumor size (cm) } \\
\hline$<4$ & $181(73.9)$ \\
\hline$\geq 4$ & $64(26.1)$ \\
\hline \multicolumn{2}{|l|}{ Grading } \\
\hline 1 & $16(6.5)$ \\
\hline 2 & $126(51.4)$ \\
\hline 3 & $103(42.1)$ \\
\hline \multicolumn{2}{|l|}{ FIGO stage } \\
\hline IB2 & $40(16.4)$ \\
\hline IIA & $21(8.6)$ \\
\hline IIB & $115(46.9)$ \\
\hline IIIA & $9(3.7)$ \\
\hline IIIB & $60(24.4)$ \\
\hline
\end{tabular}

FIGO, International Federation of Gynecology and Obstetrics.

associated with a p-value of $\leq 0.25$ were included in a multivariate survival Cox regression analysis. All reported p-values are two-sided, and p-values lower than 0.05 were considered significant.

Independently of statistical significance, we decided to include variables as nomogram parameters because non-significant variables should be assumed to have a minimal effect on survival. Nomograms were constructed to visualize the effect of each selected variable on the estimated probability. Internal validation was performed using the bootstrap method to estimate the optimism of the model. We perform bootstrap validation using 200 bootstrap datasets constructed by sampling with replacement from the original data but in such a way to maintain the same ratio of long term survivors relative to the number of patients surviving for less than 2 years (PFS) and 5 years (OS) [12].
Table 2. Histopathological characteristics

\begin{tabular}{lr} 
Characteristic & No. (\%) \\
Lymph vascular space invasion & \\
Yes & $44(18)$ \\
$\quad$ No & $201(82)$ \\
Parametria invasion & \\
$\quad$ Yes & $70(25.3)$ \\
No & $175(71.4)$ \\
Lymph node involvment & \\
Yes & $63(25.9)$ \\
No & $182(74.1)$ \\
\hline
\end{tabular}

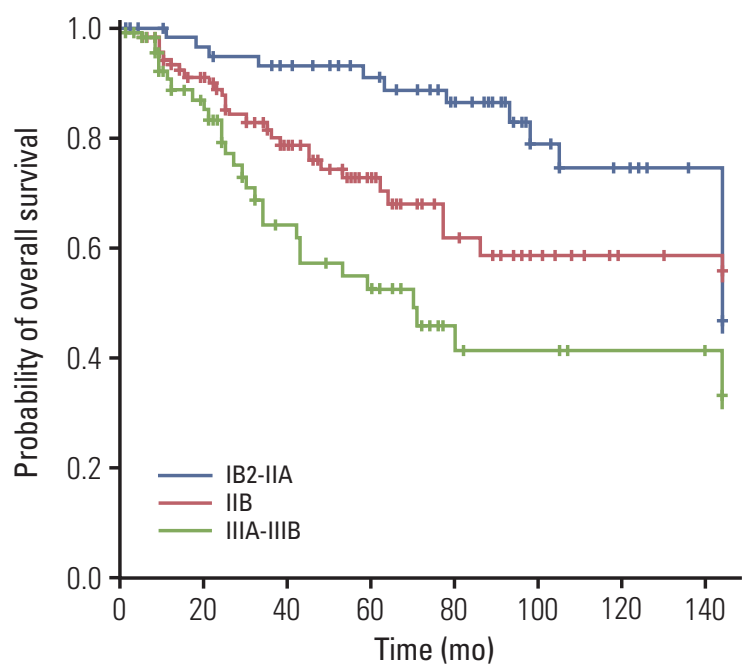

No. at risk

$\begin{array}{lrrrrrrrr}\text { IB2-IIA } & 61 & 56 & 53 & 44 & 37 & 21 & 18 & 8 \\ \text { IIB } & 115 & 86 & 61 & 45 & 22 & 19 & 19 & 5 \\ \text { IIIA-IIIB } & 69 & 47 & 31 & 23 & 10 & 10 & 10 & 5\end{array}$

Fig. 1. Overall survival according to International Federation of Gynecology and Obstetrics stage.

\section{Ethical statement}

The data were analyzed retrospectively from a database compiled prospectively, this procedure does not require the approvation by the Institutional Review Board but only the approval of the department that was obtained.

Patients have signed a consensus regarding the use of their data for scientific research. 
Table 3. Overall survival univariate and multivariate analysis

\begin{tabular}{|c|c|c|c|c|}
\hline \multirow{2}{*}{ Variable } & \multicolumn{2}{|c|}{ Univariate analysis } & \multicolumn{2}{|c|}{ Multivariate analysis } \\
\hline & Hazard ratio & p-value & Hazard ratio & p-value \\
\hline Age ( $<50$ yr vs. $\geq 50$ yr $)$ & $0.90(0.56-1.45)$ & 0.68 & - & - \\
\hline Smoker (no vs. yes) & $3.05(1.22-7.60)$ & 0.02 & $2.34(0.86-6.38)$ & 0.10 \\
\hline $\operatorname{BMI}\left(<25 \mathrm{~kg} / \mathrm{m}^{2}\right.$ vs. $\left.\geq 25 \mathrm{~kg} / \mathrm{m}^{2}\right)$ & $0.88(0.53-1.44)$ & 0.62 & - & - \\
\hline Histology (squamous vs. other) & $0.58(0.76-3.32)$ & 0.21 & $1.16(0.47-2.88)$ & 0.74 \\
\hline Tumor size $(<4 \mathrm{~cm}$ vs. $\geq 4 \mathrm{~cm})$ & $0.38(0.20-0.71)$ & $<0.01$ & $0.49(0.24-1.02)$ & 0.05 \\
\hline Grading (1-2 vs. 3$)$ & $0.63(0.39-1.01)$ & 0.06 & $0.48(0.27-0.86)$ & 0.01 \\
\hline FIGO stage (IB-II vs. III) & $0.43(0.26-0.70)$ & $<0.01$ & $0.69(0.37-1.30)$ & 0.25 \\
\hline LVSI (no vs. yes) & $0.32(0.19-0.53)$ & $<0.01$ & $0.86(0.43-1.71)$ & 0.67 \\
\hline Positive parametria (no vs. yes) & $0.21(0.13-0.35)$ & $<0.01$ & $0.43(0.19-0.97)$ & 0.04 \\
\hline Positive lymph nodes (no vs. yes) & $0.26(0.16-0.42)$ & $<0.01$ & $0.54(0.23-1.24)$ & 0.14 \\
\hline
\end{tabular}

BMI, body mass index; FIGO, International Federation of Gynecology and Obstetrics; LVSI, lymphovascular space invasion.

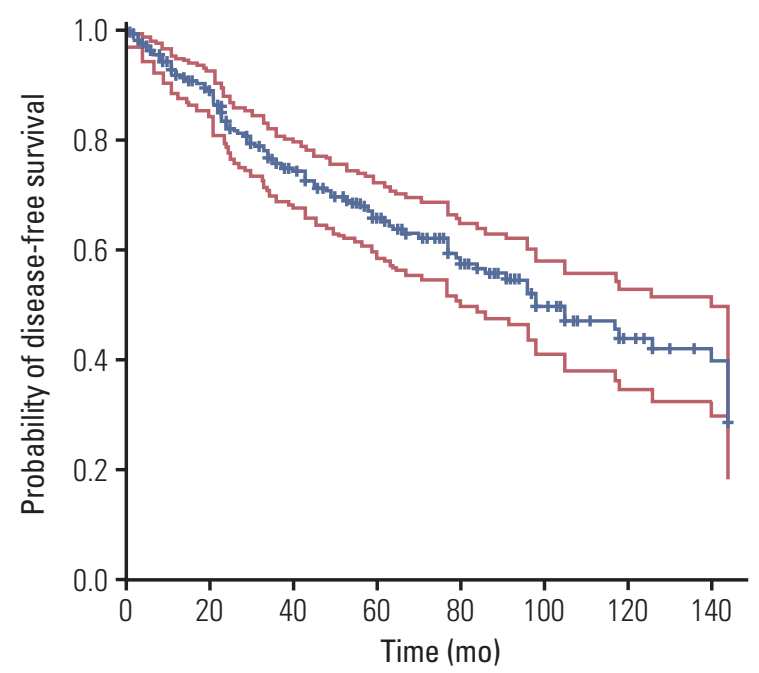

$\begin{array}{lllllllll}\text { No. at risk } & 247 & 184 & 136 & 104 & 65 & 43 & 29 & 19\end{array}$

Fig. 2. Disease-free survival for the entire study population.

\section{Results}

A total of 245 patients who underwent neoadjuvant platinum-based chemotherapy and radical surgery for primary cervical carcinoma were included in the study. The general characteristics of the patients are listed in Table 1. Mean age at diagnosis was 51 years and the vast majority of patients $(\mathrm{n}=184,75.1 \%)$ had at least tumor with parametrial invasion. Histo-pathological details are shown in Table 2.

\section{Overall survival}

The median OS was 144 months with a 5 -year OS of $72.6 \%$ (95\% confidence interval [CI], 0.66 to 0.79 ). Overall, FIGO disease stage was strongly associated with OS with a 5-year OS rates of $90.8 \%$ ( $95 \% \mathrm{CI}, 0.79$ to 0.96 ), $72.6 \%$ (95\% CI, 0.61 to 0.81 ), and $52.5 \%$ ( $95 \% \mathrm{CI}, 0.37$ to 0.66 ), for FIGO stage IB2IIA, IIB, and III, respectively ( $\mathrm{p}<0.001)$ (Fig. 1). On univariate analysis, smoke, tumor size, FIGO disease stage, lymphovascular space invasion, positive parametria, and positive lymph-nodes were significant prognostic factors associated with OS (Table 3). On multivariate analysis, tumor size, grading, and parametria status were independently prognostic (Table 2).

\section{Progression-free survival}

Median time for PFS was 98 months (range, 84 to 140 months). The 2-year and 5-year PFS were $83.7 \%$ and $66 \%$, respectively (Fig. 2). On univariate analysis, tumor size, FIGO stage, lymphovascular space invasion, parametria status and positive lymph-nodes were predictive for PFS. Age, tumor size, and positive parametria affected the rate of PFS, in multivariate analysis. Details are shown in Table 4.

\section{Nomograms}

To account for the importance of tumor and clinical pathological factors, the most predictive variables were used to create two nomograms, one to predict the 5-year probability of OS and the other to predict the 2-year probability of PFS. The concordance index of the OS nomogram was 0.66 , whereas the concordance index of the PFS nomogram was 0.64. Calibration plots demonstrated good agreement between pre- 
Table 4. Disease-free survival univariate and multivariate analysis

\begin{tabular}{|c|c|c|c|c|}
\hline \multirow{2}{*}{ Variable } & \multicolumn{2}{|c|}{ Univariate analysis } & \multicolumn{2}{|c|}{ Multivariate analysis } \\
\hline & Hazard ratio & p-value & Hazard ratio & p-value \\
\hline Age ( $<50$ yr vs. $\geq 50$ yr $)$ & $0.72(0.48-1.09)$ & 0.13 & $0.60(0.37-1.00)$ & 0.05 \\
\hline Smoker (no vs. yes) & $0.72(0.40-1.27)$ & 0.26 & - & - \\
\hline $\operatorname{BMI}\left(<25 \mathrm{~kg} / \mathrm{m}^{2}\right.$ vs. $\left.\geq 25 \mathrm{~kg} / \mathrm{m}^{2}\right)$ & $0.98(0.63-1.50)$ & 0.93 & - & - \\
\hline Histology (squamous vs. other) & $1.19(0.67-2.10)$ & 0.54 & - & - \\
\hline Tumor size $(<4 \mathrm{~cm}$ vs. $\geq 4 \mathrm{~cm})$ & $0.41(0.24-0.70)$ & $<0.01$ & $0.54(0.30-0.97)$ & 0.04 \\
\hline Grading (1-2 vs. 3$)$ & $0.33(0.10-1.06)$ & 0.07 & $0.70(0.43-1.15)$ & 0.16 \\
\hline FIGO stage (IB-II vs. III) & $0.35(0.23-0.52)$ & $<0.01$ & $0.65(0.39-1.09)$ & 0.10 \\
\hline LVSI (no vs. yes) & $0.30(0.20-0.47)$ & $<0.01$ & $0.63(0.34-1.15)$ & 0.13 \\
\hline Positive parametria (no vs. yes) & $0.28(0.18-0.42)$ & $<0.01$ & $0.44(0.22-0.86)$ & 0.02 \\
\hline Positive lymph nodes (no vs. yes) & $0.34(0.22-0.51)$ & $<0.01$ & $0.74(0.36-1.54)$ & 0.42 \\
\hline
\end{tabular}

BMI, body mass index; FIGO, International Federation of Gynecology and Obstetrics; LVSI, lymphovascular space invasion.

dicted and actual probability of OS and PFS, respectively. Fig. 3 can be used to compute the probability of 5-year OS and Fig. 4 to calculate the probability of 2-year PFS. The sum of each variable point was plotted on the total point axis, and the estimated median 5-year OS and 2-year PFS rates were respectively obtained by drawing a vertical line from the plotted total point axis straight down to the outcome axis.

To demonstrate nomograms applicability, we introduced a representative clinical case. Inside parenthesis, it is reported for each factor its OS point/PFS point, respectively. A 75-year-old woman ( 0 point $/ 0$ point) with clinical FIGO IIB (20 points $/ 5$ points) squamous cell ( 0 point $/ 0$ point) G3 (37 points/32.5 points) cervical cancer was treated at our department. Her pretreatment BMI was 22.2 (63 points/48 points). She had a primary tumor size of $4.5 \mathrm{~cm}$ (57 points / 47.5 points). She underwent NACT plus RS, without lymphovascular space invasion invasion ( 0 point $/ 0$ point), parametria invasion ( 0 point $/ 0$ point), and lymph node involvement ( 0 point $/ 0$ point). The nomograms predict a 5 -year OS of $85 \%$ and a 2-year PFS of $98 \%$. After a follow-up of 25 months, the patient is still alive, without any evidence of disease.

\section{Discussion}

The present study suggests that grading, tumor size at diagnosis, and parametrial involvement are independent prognostic factors of OS in LACC. These variables with the exception of tumor grade were predictive for PFS. Furthermore, we were able to develop a robust nomogram that can provide individual estimations of treatment outcome in this patient population.

NACT followed by radical hysterectomy is a valid alternative to concurrent CRT for patients with stage IB2-III cervical cancer; furthermore, in both randomized control trials and meta-analysis, it has been shown to be superior to radiotherapy alone and to prevent radiotherapy-related side effects $[4,5]$.

With this regard, we have previously demonstrated [13] in a relatively large population that several pathological (grading, histotype) and clinical features (FIGO stage, tumour size $>5 \mathrm{~cm}$, and parametrical involvement) strongly correlate with survival in patients submitted to this approach. To our knowledge, our nomograms represent the first attempt to develop a multifactorial prognostic model in LACC receiving NACT plus RS. First of all, it should be noticed that survival data of our series compares well with other previously published in a similar setting of women affected by LACC who received CRT instead of NACT plus RS. In fact, we obtained a 5-year OS rates of $90.8 \%, 72.6 \%$, and $52.5 \%$ for FIGO stage IB2-IIA, IIB, and III, respectively, which is similar [14] otherwise higher [15] than those recently reported, suggesting that NACT followed by RS is a feasible and effective treatment option in LACC and deserves to be further discussed and investigated.

Our analysis also demonstrates that the degree of tumor extent is highly predictive of OS and PFS. This is in accordance with our previous data [13]. As all patients received $\mathrm{NACT}$, it is easy to interpret these data as the logical consequence of the correlations between the disease volume and the tumor cell kinetics. The greater the volume, the larger the hypoxic and resting phase cell population with reduced or no chemosensitivity and the probability of developing resistant clones.

Accordingly with previous findings $[15,16]$, we have also 


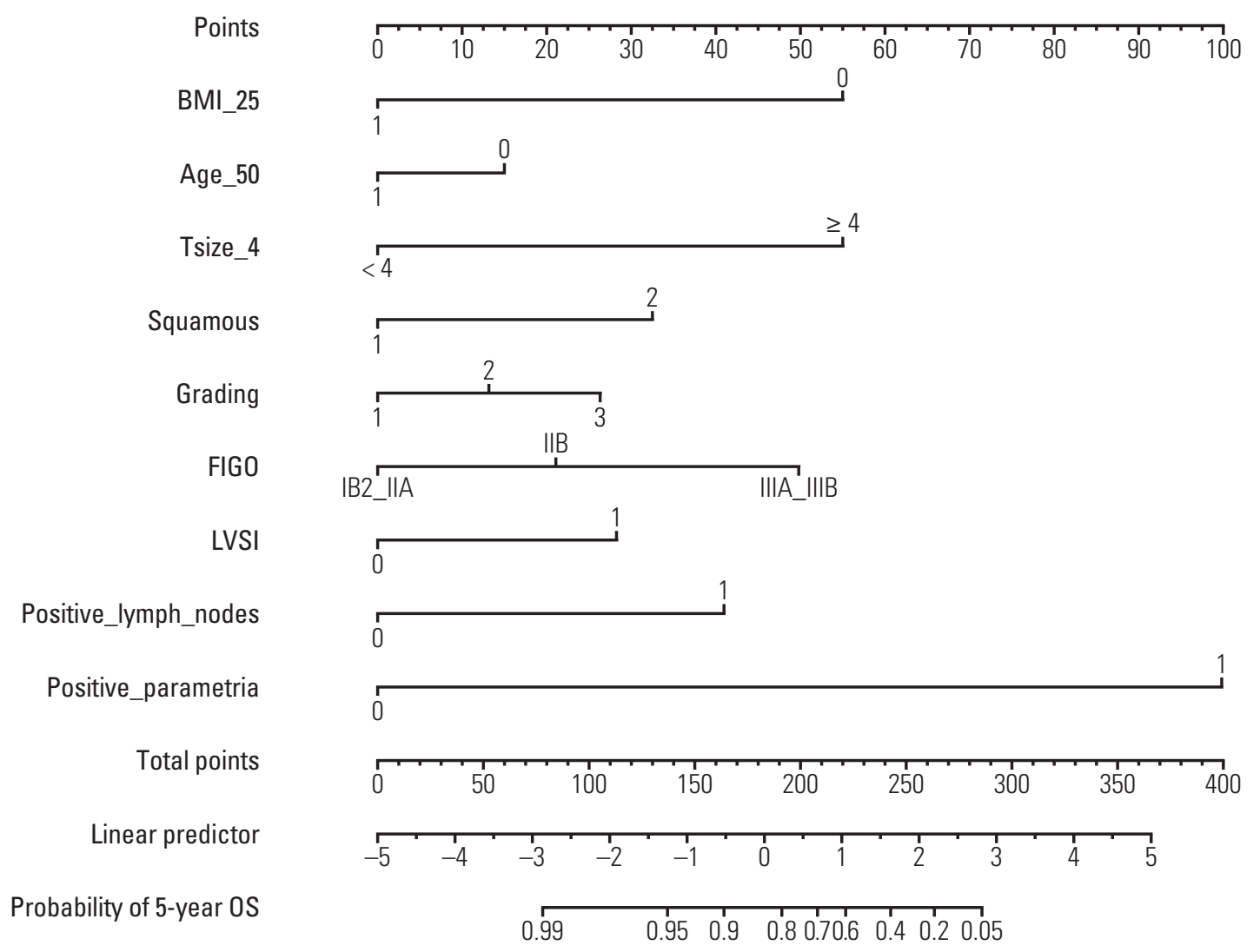

Fig. 3. Five-year overall survival nomogram. BMI 25 indicates body mass index $\geq 25$ (1) or $<25$ (0). Age_50 indicates age $\geq 50$ (1) or $<50$ (0) at diagnosis. Tsize_4 indicates clinical tumor size $\geq 4$ (1) or $<4(0)$. Squamous indicates squamous cell carcinoma (1) or other tumor histologies (2). LVSI indicates presence (1) or absence (0) of lymphovascular space invasion. Positive_lymph_nodes indicates pathological positive (1) or negative (0) locoregional lymph nodes. Positive_parametria indicates pathological positive (1) or negative (0) parametria. To use, find patient's BMI on BMI_25 axis, then draw straight line upward to points axis to determine how many points patient receives for BMI. Do this again for other axes, each time drawing straight line upward toward points axis. Sum points received for each variable, and find sum on total points axis. Draw straight line down to survival-probability axis to find patient's probability of 5-year OS. BMI, body mass index; FIGO, International Federation of Gynecology and Obstetrics; OS, overall survival.

found that parametrial involvement is a prognostic factor of both OS and PFS; besides, it appears to be predominating in the multifactorial model. Differently from other nomogram in LACC in which parametrial involvement was defined only on the basis of radiological findings [14], it should be underlined that one of the peculiarity of our study is that we only considered patients who had received RS and therefore our characterization of parametrial involvement is exclusively pathology-proven, suggesting a higher accuracy.

Regarding lymph nodal involvement, several reports found it to be an important prognostic factor $[15,16]$. In our multivariate analysis we did not confirm this finding, albeit the effect of lymph node involvement was substantial in our multifatcorial model. As stated before, it should be highlighted that all of our patients underwent pelvic with or without paraortic lymphadenectomy. This surgical approach is in contrast with nomograms developed with other cohort of patients, in which less than $37 \%$ patients underwent laparoscopic sampling of pelvic/ paraaortic lymph nodes and therefore, lymph nodal status was defined on the basis of a non-homogeneous radiological evaluation [14].

Controversial data have been proposed regarding the impact of younger age on prognosis and some authors suggested that younger age is a predictor of unfavourable prognosis, particularly in more advanced stages [17]. This was supported on the assumption that if the risk of progression 


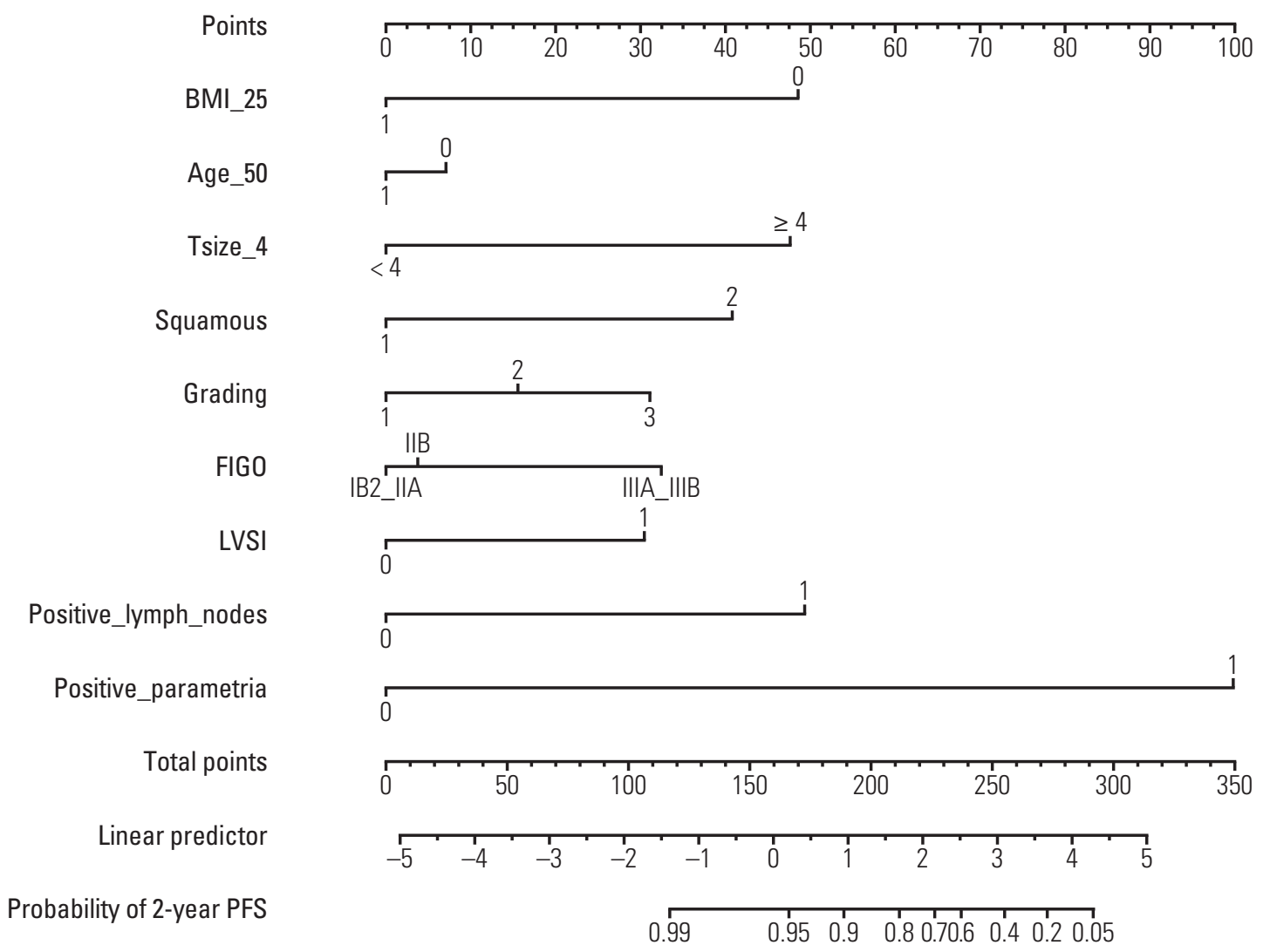

Fig. 4. Two-year progression-free survival nomogram. BMI_25 indicates body mass index $\geq 25(1)$ or $<25$ (0). Age_50 indicates age $\geq 50(1)$ or $<50(0)$ at diagnosis. Tsize_4 indicates clinical tumor size $\geq 4(1)$ or $<4(0)$. Squamous indicates squamous cell carcinoma (1) or other tumor histologies (2). LVSI indicates presence (1) or absence (0) of lymphovascular space invasion. Positive_lymph_nodes indicates pathological positive (1) or negative (0) locoregional lymph nodes. Positive_parametria indicates pathological positive (1) or negative (0) parametria. To use, find patient's BMI on BMI_25 axis, then draw straight line upward to points axis to determine how many points patient receives for BMI. Do this again for other axes, each time drawing straight line upward toward points axis. Sum points received for each variable, and find sum on total points axis. Draw straight line down to survival-probability axis to find patient's probability of 2-year PFS. BMI, body mass index; FIGO, International Federation of Gynecology and Obstetrics; PFS, progression-free survival.

from mild dysplastic changes of the cervix to severe dysplasia, let alone cancer, is only $1 \%$ per year, then the development of cancer in young women, especially the very young, must be more aggressive. In our cohort of patients, we found a slightly significant trend of younger age to be associated with disease-free survival (DFS). Therefore, we included age into our model. Nonetheless, the role of age on survival still needs to be more deeply clarified; differently, the correlation between age and response to chemotherapy seems to be stronger, suggesting that younger patients might achieve a higher response to NACT; this will be refined in further research planned in this study population (analysis ongoing, data not shown).
One limitation of our nomograms is that they are based solely on disease outcomes and do not account for the risk of treatment-related toxicity. Moreover, we are still in process to validate these nomograms on other cohort of patients to ensure their generalizability, thus at present they should be considered hypothesis generating rather than confirmatory.

Developing a nomogram for prognosis or treatment prediction has been considered helpful in individualized medicine and successful applications have been utilized in many malignancies [18-21]. This statistically based tool provides a predicted probability of a specific outcome, using a combined set of proven or potential prognostic factors. Another possible application of the nomograms could be the oppor- 
tunity to tailor follow-up schedules. Apart from early management of complications, documentation of outcomes and maintaining the patient-physician relationship, the main aim of clinical follow-up is improvement of survival. While it seems obvious that intensive follow-up improves patient outcome, there is debate about the intensity. The nomograms could assist in creating an individual follow-up schedule.

Our nomograms can assist in the decision when to treat a LACC patient treated with NACT plus RS, based on the individual risk for progression disease and death.

In summary, we constructed a nomogram based on clinical-pathological features in order to predict 2-year PFS and 5 -year OS in locally advanced cervical cancer primarily treated with NACT followed by RS. This tool might be par- ticularly helpful for assisting in the follow up of cervical cancer patients who have not undergone concurrent chemoradiotherapy. Our model indicates that parametrial involvement seems to be a major prognostic factor of both OS and DFS; besides, it appears to be predominating in the multifactorial model and thus should be systematically assessed by pathologists to help with prognostication.

To help guide decision-making, we have developed a clinically useful tool to predict the probability of both OS and PFS in LACC patients treated by NACT and RS.

\section{Conflicts of Interest}

Conflict of interest relevant to this article was not reported.

\section{References}

1. Siegel RL, Miller KD, Jemal A. Cancer statistics, 2016. CA Cancer J Clin. 2016;66:7-30.

2. Lim MC, Moon EK, Shin A, Jung KW, Won YJ, Seo SS, et al. Incidence of cervical, endometrial, and ovarian cancer in Korea, 1999-2010. J Gynecol Oncol. 2013;24:298-302.

3. National Cancer Institute. Concurrent chemoradiation for cervical cancer. Clinical announcement. Washington, DC: National Cancer Institute; 1999.

4. Neoadjuvant Chemotherapy for Locally Advanced Cervical Cancer Meta-analysis Collaboration. Neoadjuvant chemotherapy for locally advanced cervical cancer: a systematic review and meta-analysis of individual patient data from 21 randomised trials. Eur J Cancer. 2003;39: 2470-86.

5. Benedetti Panici P, Bellati F, Pastore M, Manci N, Musella A, Pauselli S, et al. An update in neoadjuvant chemotherapy in cervical cancer. Gynecol Oncol. 2007;107(1 Suppl 1):S20-2.

6. Je HU, Han S, Kim YS, Nam JH, Kim HJ, Kim JW, et al. A nomogram predicting the risks of distant metastasis following postoperative radiotherapy for uterine cervical carcinoma: a Korean radiation oncology group study (KROG 12-08). Radiother Oncol. 2014;111:437-41.

7. Kong TW, Kim J, Son JH, Kang SW, Paek J, Chun M, et al. Preoperative nomogram for prediction of microscopic parametrial infiltration in patients with FIGO stage IB cervical cancer treated with radical hysterectomy. Gynecol Oncol. 2016;142: 109-14.

8. Polterauer S, Grimm C, Hofstetter G, Concin N, Natter C, Sturdza A, et al. Nomogram prediction for overall survival of patients diagnosed with cervical cancer. Br J Cancer. 2012;107: 918-24.

9. Kidd EA, El Naqa I, Siegel BA, Dehdashti F, Grigsby PW. FDG-PET-based prognostic nomograms for locally advanced cervical cancer. Gynecol Oncol. 2012;127:136-40.

10. Seo Y, Yoo SY, Kim MS, Yang KM, Yoo HJ, Kim JH, et al.
Nomogram prediction of overall survival after curative irradiation for uterine cervical cancer. Int J Radiat Oncol Biol Phys. 2011;79:782-7.

11. Edge S, Byrd DR, Compton CC, Fritz AG, Greene FL, Trotti A. AJCC cancer staging handbook. New York: Springer-Verlag; 2010.

12. Capanu M, Gonen M. Building a nomogram for surveyweighted Cox models using R. J Stat Softw. 2015;64:1-17.

13. Benedetti-Panici P, Greggi S, Scambia G, Amoroso M, Salerno MG, Maneschi F, et al. Long-term survival following neoadjuvant chemotherapy and radical surgery in locally advanced cervical cancer. Eur J Cancer. 1998;34:341-6.

14. Rose PG, Java J, Whitney CW, Stehman FB, Lanciano R, Thomas GM, et al. Nomograms predicting progression-free survival, overall survival, and pelvic recurrence in locally advanced cervical cancer developed from an analysis of identifiable prognostic factors in patients from NRG Oncology / Gynecologic Oncology Group randomized trials of chemoradiotherapy. J Clin Oncol. 2015;33:2136-42.

15. Tseng JY, Yen MS, Twu NF, Lai CR, Horng HC, Tseng CC, et al. Prognostic nomogram for overall survival in stage IIB-IVA cervical cancer patients treated with concurrent chemoradiotherapy. Am J Obstet Gynecol. 2010;202:174.e1-7.

16. Benedetti-Panici P, Greggi S, Colombo A, Amoroso M, Smaniotto D, Giannarelli D, et al. Neoadjuvant chemotherapy and radical surgery versus exclusive radiotherapy in locally advanced squamous cell cervical cancer: results from the Italian multicenter randomized study. J Clin Oncol. 2002;20: 179-88.

17. Chen RJ, Lin YH, Chen CA, Huang SC, Chow SN, Hsieh CY. Influence of histologic type and age on survival rates for invasive cervical carcinoma in Taiwan. Gynecol Oncol. 1999; 73:184-90.

18. Gronchi A, Miceli R, Shurell E, Eilber FC, Eilber FR, Anaya 
DA, et al. Outcome prediction in primary resected retroperitoneal soft tissue sarcoma: histology-specific overall survival and disease-free survival nomograms built on major sarcoma center data sets. J Clin Oncol. 2013;31:1649-55.

19. Meretoja TJ, Strien L, Heikkila PS, Leidenius MH. A simple nomogram to evaluate the risk of nonsentinel node metastases in breast cancer patients with minimal sentinel node involvement. Ann Surg Oncol. 2012;19:567-76.
20. Wang Y, Li J, Xia Y, Gong R, Wang K, Yan Z, et al. Prognostic nomogram for intrahepatic cholangiocarcinoma after partial hepatectomy. J Clin Oncol. 2013;31:1188-95.

21. Giordano A, Egleston BL, Hajage D, Bland J, Hortobagyi GN, Reuben JM, et al. Establishment and validation of circulating tumor cell-based prognostic nomograms in first-line metastatic breast cancer patients. Clin Cancer Res. 2013;19:1596-602. 